Mitochondrial DNA-enriched

\title{
microparticles promote acute-on-chronic alcoholic neutrophilia and hepatotoxicity
}

\author{
Yan Cai, ${ }^{1}$ Ming-Jiang Xu, ${ }^{1}$ Erik H. Koritzinsky, ${ }^{2}$ Zhou Zhou, ${ }^{1}$ Wei Wang, ${ }^{1}$ Haixia Cao, ${ }^{1}$ Peter S.T. Yuen, ${ }^{2}$ \\ Ruth A. Ross, ${ }^{3}$ Robert A. Star, ${ }^{2}$ Suthat Liangpunsakul, ${ }^{3,4}$ and Bin Gao ${ }^{1}$ \\ 'Laboratory of Liver Diseases, National Institute on Alcohol Abuse and Alcoholism (NIAAA), ${ }^{2}$ Renal Diagnostics and \\ Therapeutics Unit, National Institute of Diabetes and Digestive and Kidney Diseases, NIH, Bethesda, Maryland, \\ USA. 'BDivision of Gastroenterology and Hepatology, Department of Medicine, Indiana University School of Medicine, \\ Indianapolis, Indiana, USA. ${ }^{4}$ Roudebush Veterans Administration Medical Center, Indianapolis, Indiana, USA.
}

Over the last several years, one of the major advances in the field of alcoholic liver disease research was the discovery that binge alcohol consumption induced neutrophilia and hepatic neutrophil infiltration in chronically ethanol-fed mice and human subjects with excessive alcohol use (EAU); however, the underlying mechanisms remain obscure. Here, we demonstrated that chronic EAU patients with a history of recent excessive drinking (EAU + RD) had higher serum levels of mitochondrial DNA (mtDNA)-enriched microparticles (MPs) than EAU without recent drinking (EAU - RD) and healthy controls, which correlated positively with circulating neutrophils. Similarly, mice with chronic-plus-binge (E10d + 1B) ethanol feeding also had markedly elevated serum levels of mtDNA-enriched MPs, with activation of hepatic ER stress and inflammatory responses. Inhibition of ER stress by gene $\mathrm{KO}$ or inhibitors attenuated ethanol-induced elevation of mtDNA-enriched MPs, neutrophilia, and liver injury. The data from the study of hepatocyte-specific deletion of the protein kinase RNA-like ER kinase (Perk) gene in mice and of cultured hepatocytes demonstrated that hepatocytes were the main source of mtDNA-enriched MPs after ethanol feeding. Finally, administration of mtDNA-enriched MPs isolated from E10d+1B-fed mice caused neutrophilia in mice. In conclusion, E10d + 1B ethanol consumption activates hepatic ER stress-dependent mtDNAenriched MP release, leading to neutrophilia and liver injury.

Authorship note: $\mathrm{YC}$ and $\mathrm{MJX}$ contributed equally to this work.

Conflict of interest: The authors have declared that no conflict of interest exists.

Submitted: January 4, 2017 Accepted: June 13, 2017 Published: July 20, 2017

\section{Reference information:} JCI Insight. 2017;2(14):e92634 https://doi.org/10.1172/jici. insight.92634.

\section{Introduction}

Excessive alcohol consumption is a leading cause of chronic liver disease and contributes to approximately half of liver cirrhosis in Western countries $(1,2)$. Despite the massive economic and health burden of alcoholic cirrhosis, little development in therapeutic strategy has been made for patients with this severe malady due to incomplete understanding in the mechanism and pathogenesis of alcoholic liver disease (ALD) $(1,3)$. ALD represents a broad spectrum of hepatic pathology ranging from simple steatosis (fatty liver) to severe forms of liver injury, such as steatohepatitis, fibrosis/cirrhosis, and hepatocellular carcinoma (HCC) (1). The earliest response of the liver to excessive alcohol drinking is the accumulation of fat in the hepatocytes, which occurs in almost all heavy drinkers. Interestingly, only $20 \%-40 \%$ of heavy drinkers develop steatohepatitis, which is characterized by infiltration of neutrophils and hepatic inflammation in the presence of steatosis. Once developed, it can progress to cirrhosis and HCC (4). However, the mechanisms underlying hepatic neutrophil infiltration in alcoholics are not fully understood. Recently, we reported that chronic excessive alcohol use (EAU) patients with recent excessive drinking (RD) $(E A U+R D)$ had much higher levels of circulating neutrophils, which positively correlated with elevated serum levels of alanine transaminase (ALT) and aspartate transaminases (AST) when compared with those without RD (EAU - RD) and healthy controls (5). These clinical data suggest that RD (or binge drinking) in patients with chronic EAU elevates peripheral blood neutrophils and likely leads to hepatic neutrophil infiltration and liver injury. Binge drinking-mediated elevation of circulating neutrophils was also demonstrated in several mouse models. For example, acute ethanol gavage markedly elevated circulating and hepatic neutrophils in chronically 
ethanol-fed mice or in high-fat diet-fed mice $(6,7)$. In addition, weekly ethanol binge also caused significant hepatic neutrophil infiltration in chronically intragastric ethanol-fed mice (8). However, the mechanisms by which binge drinking causes neutrophilia are not fully understood.

Chemotaxis driven by a variety of chemokines is the first step in recruiting neutrophils. Among these chemokines, IL-8 is one of the most important chemokines for neutrophil recruitment in humans (9). It has been reported that serum IL-8 levels are highly elevated and positively correlate with increased circulating and hepatic neutrophil numbers, along with disease severity in patients with alcoholic hepatitis (10-12). CXCL1, a functional homologue of human IL-8, plays a key role in promoting neutrophil recruitment in mice. Several studies have demonstrated that hepatic and serum CXCL1 levels are elevated and contribute to the liver injury in several mouse models of $\operatorname{ALD}(7,13)$. Blockage of the CXCL1 receptor CXCR1/2 ameliorated alcoholic steatohepatitis in mice (14). In addition to chemokines, extracellular vesicles (EVs) are new players in cellto-cell communication and play an important role in promoting inflammation and neutrophil infiltration in a variety of diseases $(15,16)$, including liver diseases (17-25). EVs are nanometer-sized, membrane-bound extracellular milieu vesicles derived from cells (17). Based on their cellular biogenesis, EVs can be divided into 3 groups based on their sizes; exosomes (40-150 nm diameter in size), microvesicles/microparticles (MPs, $50-1,000 \mathrm{~nm}$ diameter), and apoptotic bodies (>500 $\mathrm{nm}$ in diameter) (17). The functions of EVs are mediated by delivering their contents from one cell to another. For example, stressed hepatocytes can activate macrophages via the release of EVs that contain lipids, proteins, chemokines, and nucleic acids (e.g., mitochondrial DNA [mtDNA]) (17-25). However, whether EVs also contribute to the elevation of circulating and hepatic neutrophils induced by acute-on-chronic alcoholic liver injury in human and mice remains unknown.

In the current study, we found that the levels of circulating EVs and MPs were much higher in EAU + $\mathrm{RD}$ compared with those in EAU - RD patients and healthy controls and correlated positively with serum levels of aminotransferases, circulating neutrophils, and serum MP mtDNA contents. To understand the mechanisms by which EVs are released after acute-on-chronic liver injury, we performed chronic-plusbinge $(\mathrm{E} 10 \mathrm{~d}+1 \mathrm{~B})$ ethanol feeding in mice, in which we found that the levels of circulating MPs were also markedly elevated after E10d $+1 B$ ethanol feeding and that hepatic ER stress contributed to such elevation. Additionally, mtDNA-enriched MPs from ethanol-fed mice promoted neutrophilia and liver injury via the activation of TLR9, thereby inducing liver injury.

\section{Results}

Serum MP mtDNA contents are elevated and positively correlate with the levels of blood neutrophils in $E A U+R D$ patients. Our recent human data showed that EAU $+\mathrm{RD}$ patients had much higher levels of circulating neutrophils, which positively correlated with elevated serum levels of ALT and AST (5). To further explore the underlying mechanism of neutrophilia, particularly in those with recent excessive alcohol drinking, we analyzed serum EVs, MPs, and mtDNA content in 171 EAU patients in whom serum samples were available. In agreement with our previous data (5), the levels of circulating neutrophils were higher among 171 EAU subjects when compared with healthy controls, which positively correlated with serum ALT levels (Supplemental Figure 1, A and B; supplemental material available online with this article; https://doi.org/10.1172/jci.insight.92634DS1).

Next, we quantified the levels of circulating particles and mtDNA contents. As illustrated in Figure 1, A and B, the serum levels of particles were higher in EAU + RD patients compared with those in controls and EAU - RD. A previous study reported the presence of plasma mtDNA within MPs in mice and patients with nonalcoholic steatohepatitis (18). We therefore purified exosomes and MPs from human sera and examined whether mtDNA is located either in the MPs or exosomes in these subjects. Here, we found that both genomic DNA (Supplemental Figure 1C) and cytochrome $c$ oxidase subunit III (CYTO $C O X$ ) mtDNA (Figure 1C) were mainly detected in MPs, whereas exosomes only contained very low levels of genomic DNA and Cyto c ox mtDNA. Based on these results, the levels of mtDNA were only measured in MPs in all subsequent experiments. As illustrated in Figure 1D, MPs from EAU + RD had much higher levels of mtDNA than those from healthy controls and EAU - RD patients, while total DNA content was comparable among the 3 groups (Supplemental Figure 1D).

Because mtDNA is known to promote neutrophil recruitment (26), we analyzed the correlation of mtDNA with circulating neutrophils. As illustrated in Figure 1, E and F, the percentage of PMN and the levels of circulating neutrophils positively correlated with the content of CYTO COX mtDNA in MPs in all subjects in our study cohort. To further analyze the correlations among groups, we found that the positive correlation was observed in EAU + RD subjects but not in EAU - RD and controls (Figure 1G). 
A
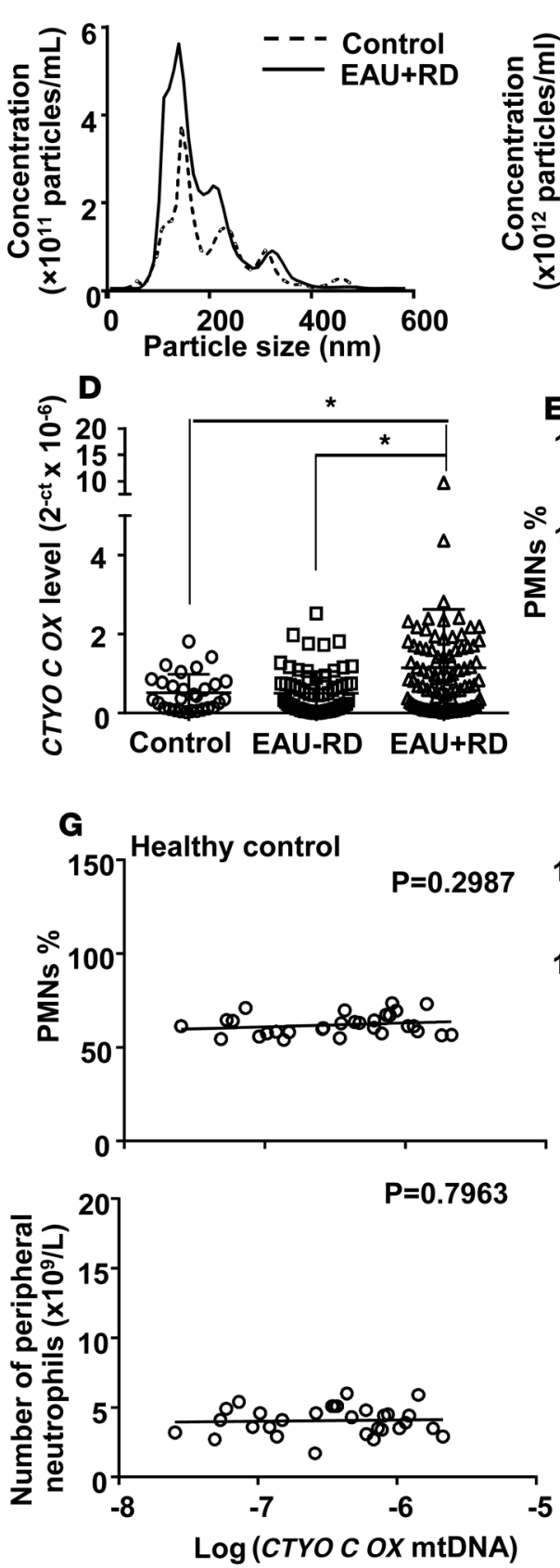
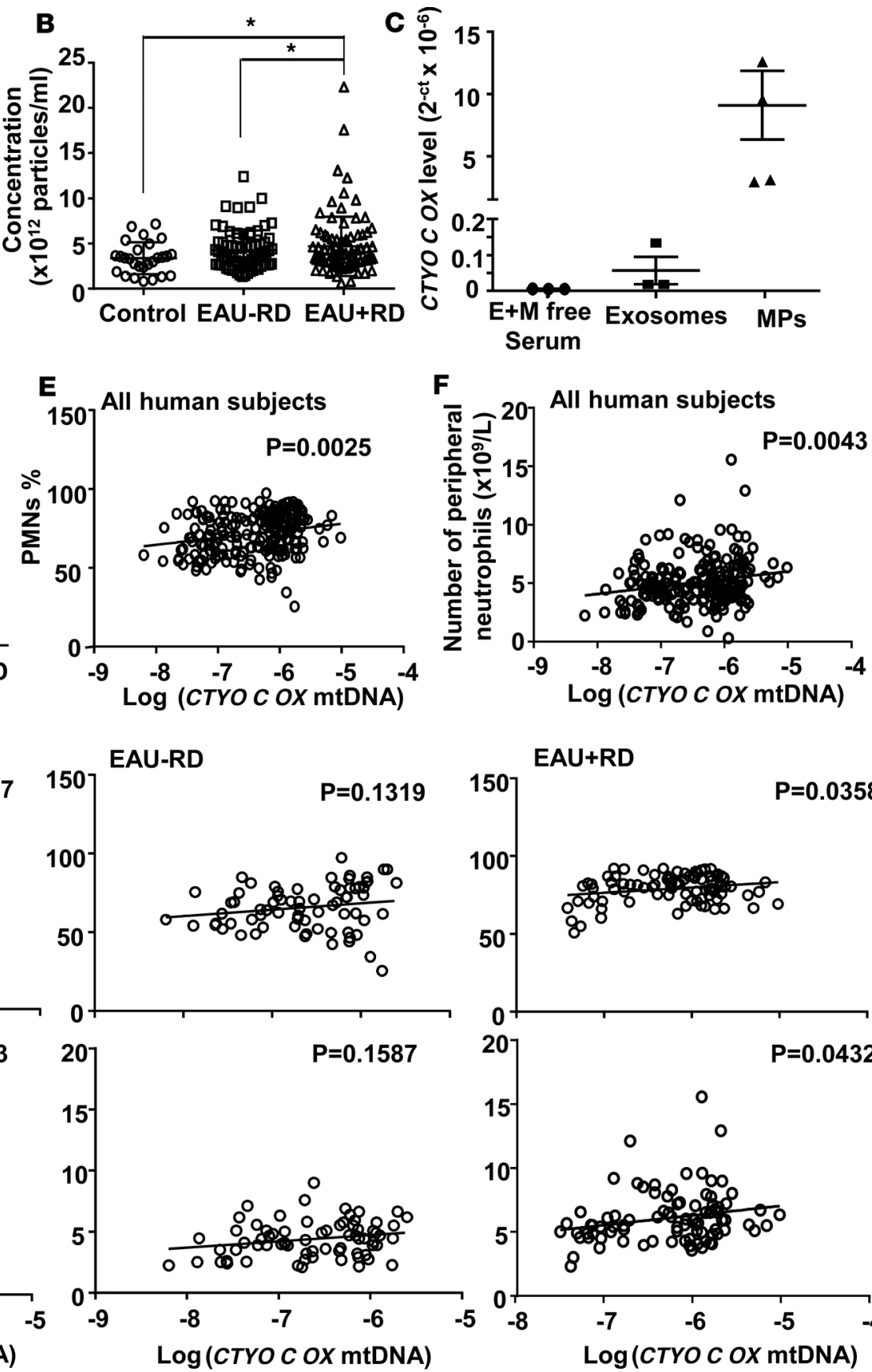

EAU+RD

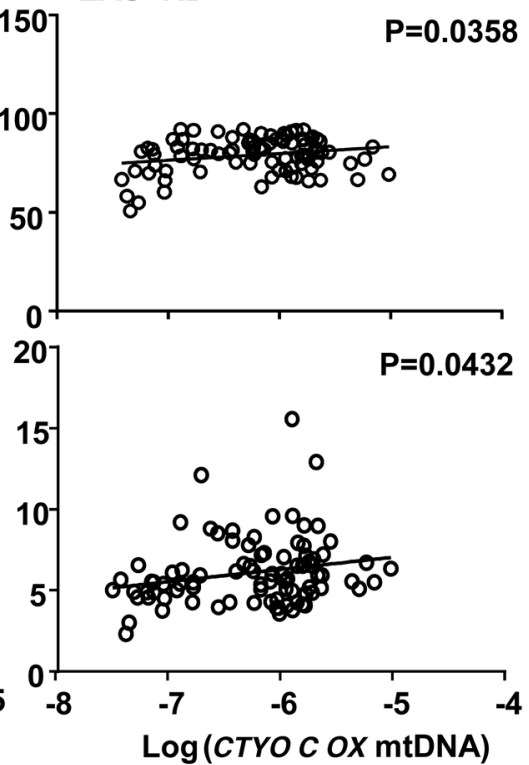

Figure 1. Serum MP mtDNA levels correlate with the levels of circulating neutrophils in patients with excessive alcohol use (EAU). (A) Representative image of the number and diameter $(\mathrm{nm})$ of the serum particles identified by using an NTA system. $n=4$. (B) The total numbers of particles in the sera of healthy control human (control, $n=30)$, EAU - RD ( $n=71)$, and EAU + RD $(n=100)$ patients were counted using an NTA system. (C) MPs and exosomes were isolated from human sera, $\mathrm{E}+\mathrm{M}$-free sera were also collected. Then, total DNA was extracted. The content of mtDNA was assessed by the measurement of CYTO C OX gene copies. (D) The levels of CYTO C OX DNA in MPs from different groups are shown. (E and F) Correlation analyses between CYTO C OX DNA in MPs and the percentage and total number of circulating neutrophils in all subjects. (C) Correlation analyses between CYTO C OX DNA in MPs and the percentage and total number of circulating neutrophils in different groups. Ordinary 1-way ANOVA with Tukey's test was performed in B and $\mathbf{D}$. ${ }^{*} P<0.05$. Linear regression analyses were performed in $\mathbf{E}-\mathbf{G}$, and $P$ values are shown in the figures.

E10d $+1 B$ ethanol feeding induces neutrophilia and increases serum mtDNA and MPs. The above human data suggest that recent excessive alcohol (binge) drinking leads to the elevation in the levels of serum mtDNA and circulating neutrophils. To explore the underlying mechanisms, we used a E10d $+1 \mathrm{~B}$ ethanol feeding mouse model, which mimics the EAU + RD pattern in humans (5). As illustrated in Figure $2 \mathrm{~A}$, the number and percentage of circulating neutrophils were also elevated in the E10d $+1 \mathrm{~B}$ group 

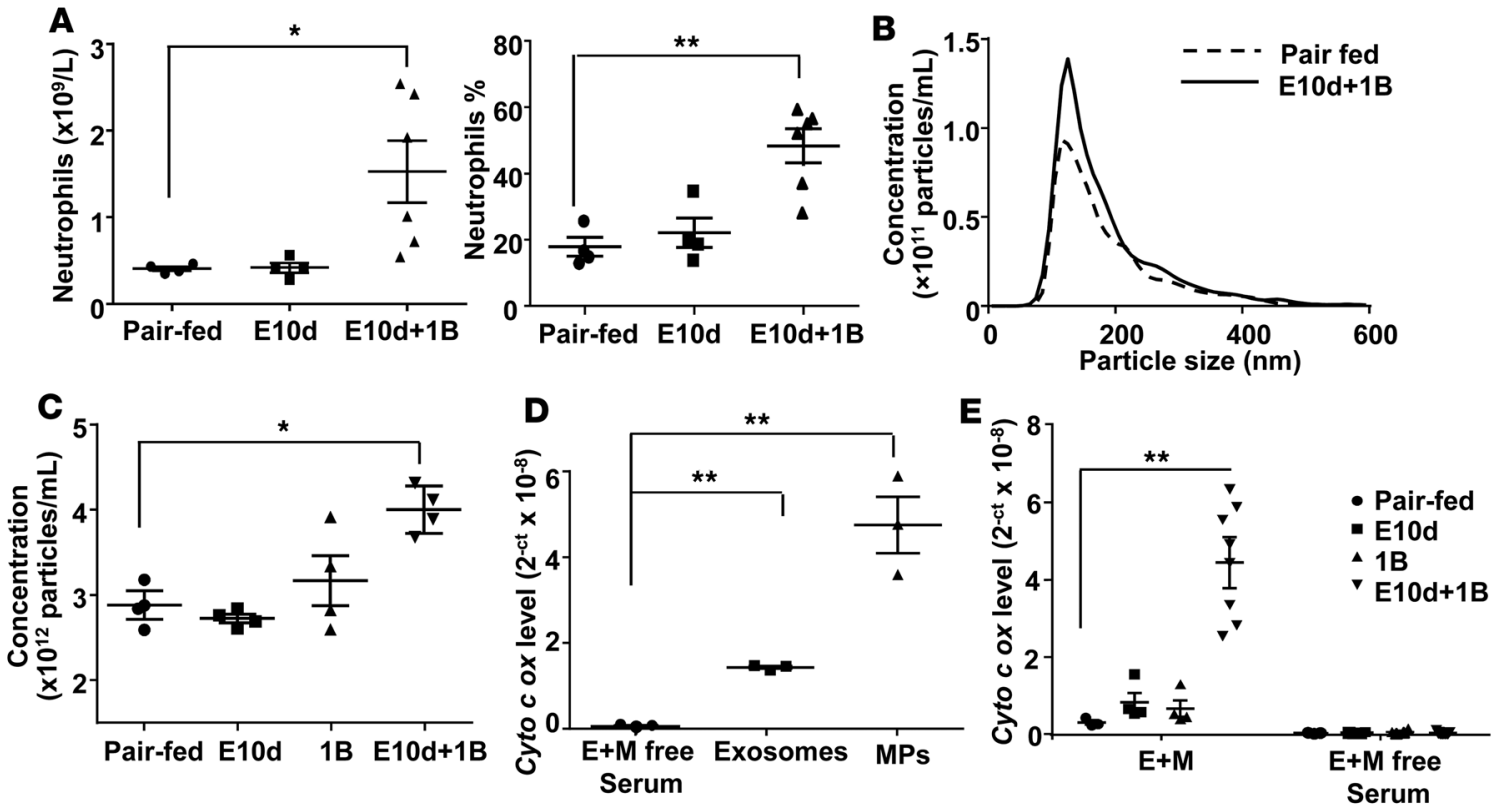

Figure 2. Chronic-plus-binge ethanol feeding (E10d + 1B) induces neutrophilia and increases serum mtDNA in MPs. (A) Mice were subjected to pair-fed $(n=4)$, E10d $(n=4)$, and E10d $+1 B(n=6)$. The number and percentage of peripheral neutrophils were measured. (B) The number and diameter $(n m)$ of the serum particles were identified by using an NTA system. (C) The number of serum particles was calculated $(n=4)$. (D) The contents of Cyto $c$ ox DNA in serum exosomes and MPs, and E + M-free serum from E10+1B mice, were analyzed $(n=3)$. (E) Mice were subjected to pair-fed $(n=4)$, E10d $(n=4)$, E10d + $1 \mathrm{~B}(n=6)$, or acute ethanol gavage $(n=4)$ alone (1B). The content of Cyto c ox DNA in the serum E + M and E + M-free sera were measured. Ordinary 1-way or 2-way (E) ANOVA with Tukey's test was performed. ${ }^{*} P<0.05,{ }^{* *} P<0.01$.

compared with pair-fed controls and chronic alcohol feeding alone (E10d). By using the nanoparticle tracking analysis (NTA), we found that the number of serum particles was higher in E10d $+1 \mathrm{~B}$ group compared with pair-fed, E10d and 1B mice (Figure 2, B and C).

Furthermore, we measured Cyto c ox DNA in the exosomes, MPs, and exosomes + MP-free (E + Mfree) serum, and found that mtDNA was mainly detected in MPs, similar to the results in human subjects (Figure 2D). Finally, Figure 2E shows that $\mathrm{mtDNA}$ levels in $\mathrm{E}+\mathrm{M}$ particle mixtures were higher in $\mathrm{E} 10 \mathrm{~d}$ $+1 \mathrm{~B}$ mice compared with pair-fed, E10d, and 1B mice.

E10d $+1 B$ ethanol feeding synergistically induces hepatic ER stress. Because it has been reported that chronic ethanol feeding induces hepatic ER stress (27-29), and ER stress promotes hepatocytes to release EVs (30), we asked whether elevation of serum MPs after E10d + 1B ethanol feeding was also due to the induction of hepatic ER stress.

Electron microscopy analyses revealed obvious ER dilation in the livers from E10d $+1 \mathrm{~B}$ ethanol-treated mice but not in pair-fed, E10d-, or 1B-treated mice (Figure 3A). To determine the presence of ER stress, we examined hepatic expression of ER stress-related genes and proteins. As illustrated in Figure 3B, hepatic expression of several ER stress-related genes, including Chop, Erola, Gadd34, Bip, Bim, $B a k$, and $D r 5$, were significantly upregulated in E10d + 1B-teated mice compared with pair-fed controls. In contrast, no changes in hepatic expression of these genes were observed in E10d- and 1B-fed mice, except hepatic Bip mRNA was elevated in 1B-treated mice (Figure 3C). Moreover, Western blot analyses revealed that hepatic expression of several ER stress-associated proteins was markedly upregulated after E10d $+1 B$ treatment (Figure 3D). These proteins include phosphorylation of the eukaryotic initiation factor $2 \alpha$ subunit (P-eIF2 $\alpha$ ), transcription factor C/EBP homologous protein (CHOP), and phosphorylation of Jun-amino-terminal kinase (P-JNK). Expression of these proteins was elevated as early as 3 hours after gavage and reached the peak levels 9 hours after gavage (Figure 3D).

Increased hepatic $\mathrm{CHOP}$ protein expression in $\mathrm{E} 10 \mathrm{~d}+1 \mathrm{~B}$-treated mice was further determined by immunostaining. As illustrated in supplemental Figure 2, no $\mathrm{CHOP}^{+}$hepatocytes were detected in pairfed or 1B-fed mice. A few weakly $\mathrm{CHOP}^{+}$staining hepatocytes were also observed around the central vein in the livers from E10d-fed mice. Interestingly, strong $\mathrm{CHOP}^{+}$stained hepatocytes around the central vein were frequently observed in mice receiving E10d $+1 \mathrm{~B}$ treatment. 
A

Pair-fed

1B

E10d

$E 10 d+1 B$
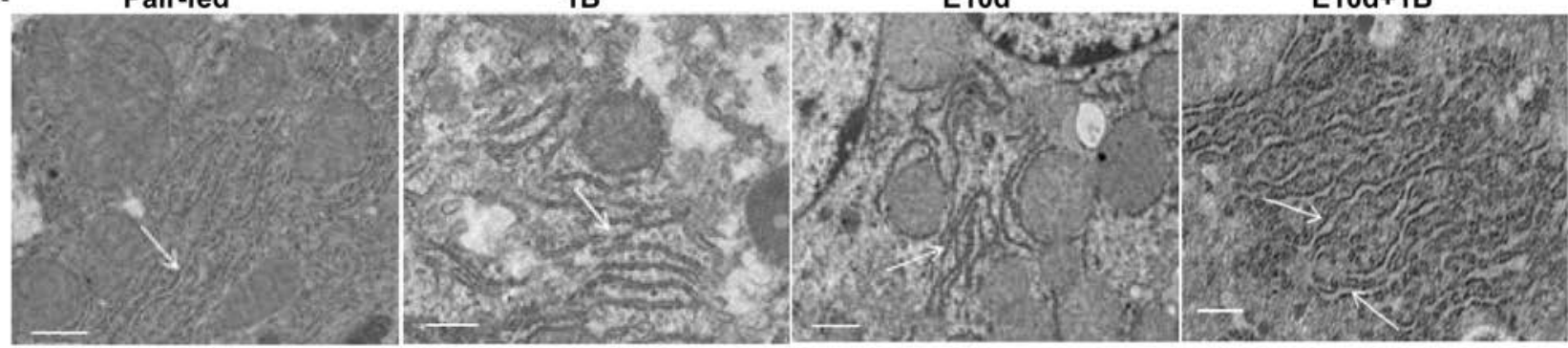

B

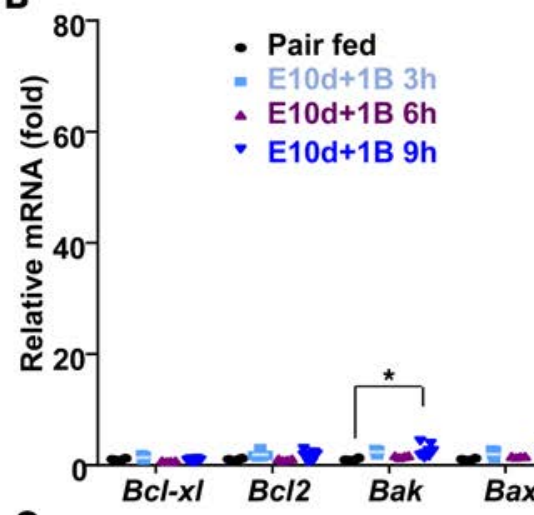

- Pair fed

- E10d+1B 3h

C

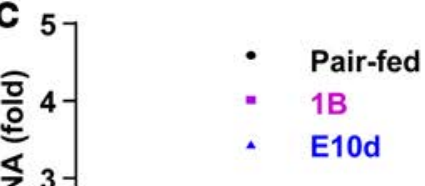<smiles>CCCCC1CCCCC1C</smiles>

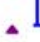

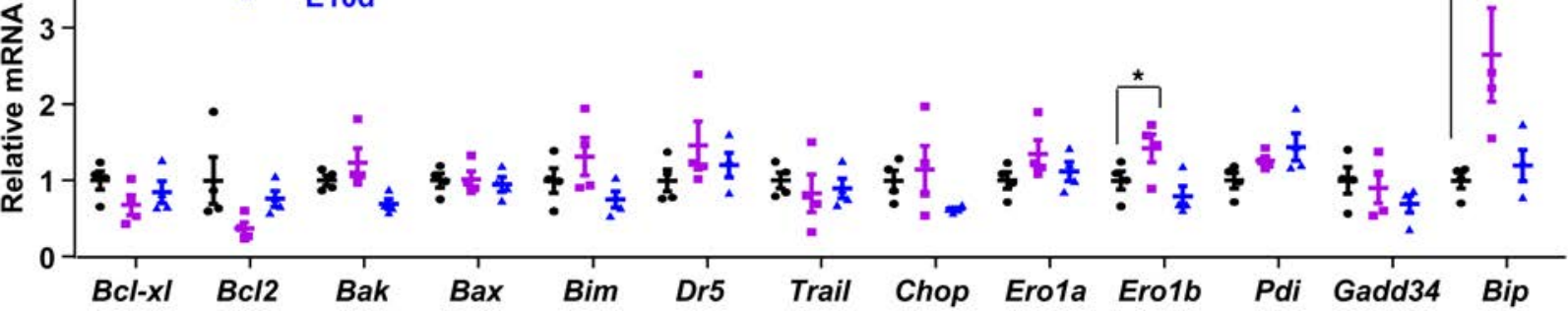
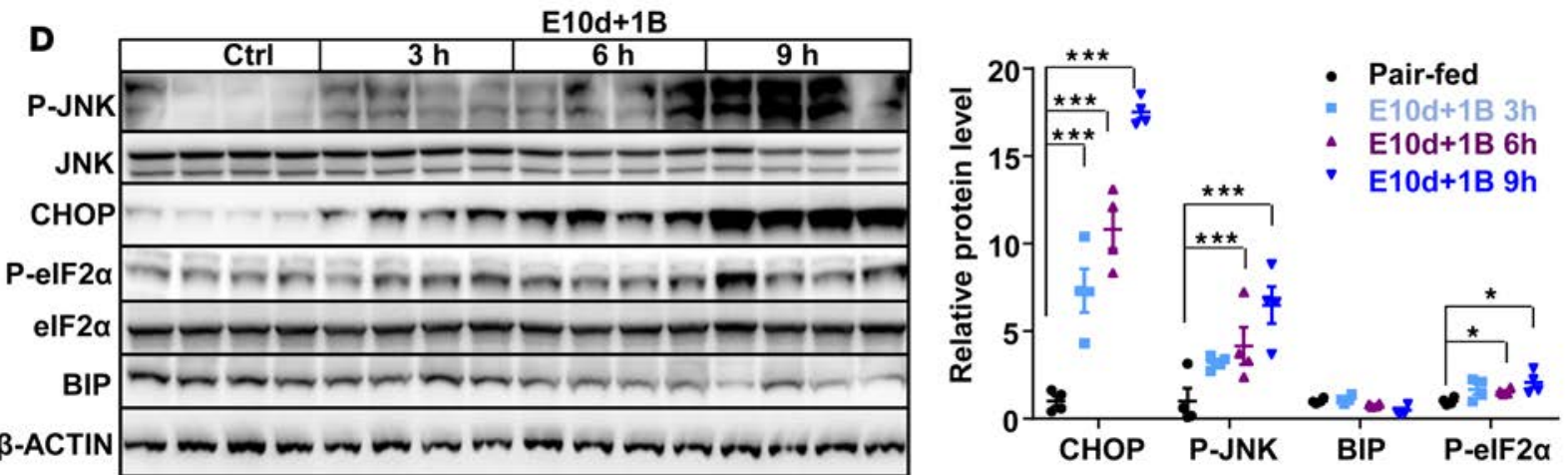

Figure 3. Chronic-plus-binge ethanol feeding synergistically induces hepatic ER stress. (A) Electron microscopy images of the liver from pair-fed, 1B, E10d, and E10d + 1B-treated mice. Arrows indicate the endoplasmic reticulum (ER). Note: substantial ER dilation is observed in E10d $+1 B$ group. Scale bar: $50 \mathrm{~nm}$. (B and C) Real-time PCR analyses of mitochondrial apoptosis and ER stress-related genes in the liver ( $n=4-6$ per group). (D) ER stress signaling-related proteins were detected by Western blot (left panel) and quantified (right panel) $(n=4)$. Ordinary 1-way ANOVA with Tukey's test was performed within each gene. ${ }^{*} P<0.05,{ }^{* *} P<0.01,{ }^{* * *} P<0.001$. 
E10d $+1 B$ ethanol feeding induces ER stress and liver injury dependent on JNK2 activation. The above data show that hepatic ER stress and JNK are activated after E10d + 1B ethanol feeding. To further confirm the role of ER stress and JNK signaling in E10d + 1B-induced liver injury, E10d + 1B-fed C57BL/6J mice were treated with ER stress inhibitors (4-phenylbutyric acid [PBA] and tauroursodeoxycholic acid [TUDCA]) or total JNK inhibitor (JNK inhibitor II). As shown in Figure 4A, hepatic mRNA expression levels of mitochondrial apoptosis pathway (Bak, Bim) and ER stress-related genes (Chop, Ero1a, Gadd34) were lower in mice treated with PBA, TUDCA, or JNK inhibitor II compared with those treated with vehicle. Moreover, treatment with PBA, TUDCA, and JNK inhibitor II ameliorated hepatic injury as demonstrated by a significant reduction of serum levels of ALT (Figure 4B) and a significant reduction of hepatic neutrophil infiltration (Figure 4C).

JNK includes two major isoforms JNK1 and JNK2, and these two isoforms play many important roles in the pathogenesis of liver diseases (31). To further dissect the role of JNK1 and JNK2 in E10d $+1 \mathrm{~B}$-induced ER stress and injury, E10d + 1B ethanol feeding experiments were performed in $J n k 1^{-1-}$ and $J n k 2^{-1-}$ mice, as well in $C h o p^{-/-}$mice. As illustrated in Figure 4D, serum levels of ALT and AST were significantly lower in $\mathrm{Jnk}^{-{ }^{--}}$and $\mathrm{Chop}^{-/-}$mice than in WT mice. Serum levels of ALT and AST were also lower in $J n k 1^{-/-}$mice compared with WT mice, but this difference did not reach statistical significance. $J n k 1^{-/-}$and $J n k 2^{-/-}$mice had comparable levels of liver steatosis while $C_{h o p^{-/}}$mice exhibited increased liver steatosis compared with WT mice (Figure 4, E and F). In agreement with these findings, hepatic expression of CHOP protein was also markedly decreased in $J n k 2^{-/-}$mice but not in $J n k 1^{-/-}$mice (Figure $4 \mathrm{G}$ ), suggesting that CHOP is a major downstream target of JNK signaling. Moreover, hepatic expressions of Bim, Dr5, Chop, Erola, and Gadd34 mRNAs were lower in E10d + 1B-treated $J n k 2^{-1-}$ mice compared with WT mice (Figure $4 \mathrm{H}$ ).

ER stress mediates E10d $+1 B$ ethanol feeding-increased serum particles, mtDNA in particles, and neutrophil infitration. Next, we examined whether ER stress contributes to the elevation of serum particles and mtDNA after E10d + 1B feeding. As illustrated in Figure 5A, serum levels of particles in $\mathrm{Jnk}^{-{ }^{--}}$and Chop ${ }^{-/-}$mice were significantly lower than those in WT mice after E10d $+1 \mathrm{~B}$ feeding, while comparable levels of serum particles were found in $J n k 1^{1_{-}-}$mice and WT mice. Because $J n k 2^{-1-}$ mice but not $J n k 1^{-_{-}-}$mice had significantly lower levels of liver injury (Figure 4D) and serum particles (Figure 5A) than WT mice, we only examined $J n k 2^{-/-}$mice but not $J n k 1^{-/-}$mice in the rest of studies.

Figure 5B shows that mtDNA contents in serum particles including exosomes and MPs from $J_{n k 2^{-/}}$and Chop $^{-1-}$ mice were significantly lower than those in WT mice after E10d $+1 \mathrm{~B}$ feeding. The number of $\mathrm{MPO}^{+}$ neutrophils in the liver was significantly elevated in WT mice after E10d $+1 \mathrm{~B}$ challenge, and this elevation was blunted in $\mathrm{Jnk}^{-{ }^{--}}$and Chop ${ }^{-/-}$mice (Figure 5C). In addition, circulating neutrophil numbers and percentage were markedly reduced in E10d $+1 \mathrm{~B}$-treated $\mathrm{Chop}^{-/-}$mice compared with WT mice (Figure 5D).

$E R$ stress promotes particle release after E10d $+1 B$ feeding dependent on hepatic inflammasome component caspase-1 activation. Activation of the inflammasome has been shown to contribute to alcoholic steatohepatitis in mice after chronic alcohol feeding (32) or E10d + 1B ethanol feeding (33), and activation of inflammasome component caspase-1 promotes particle release (34). We hypothesized that ER stress promotes particle release via the activation of the inflammasome in mice fed with E10d $+1 \mathrm{~B}$. As illustrated in Figure $6 \mathrm{~A}, \mathrm{E} 10 \mathrm{~d}+1 \mathrm{~B}$ feeding markedly upregulated hepatic expression of inflammasome-associated genes (Casp1, Nlrp3, and Il1b). Expression of pro-CASP1 protein was downregulated, while cleaved caspase-1 protein (p20) was upregulated after E10d $+1 \mathrm{~B}$ (Figure 6B), suggesting caspase 1 is activated. Hepatic expression of Casp1, Nlrp3, and Illb mRNAs was lower in $J n k 2^{-1-}$ and Chop ${ }^{-1-}$ mice compared with WT mice after E10d $+1 B$ (Figure 6C). In addition, expression of pro-CASP1 and cleaved CASP1 p20 was lower in ethanol-fed $J_{n k 2^{-1-}}$ and $\mathrm{Chop}^{-/-}$mice than in ethanol-fed WT mice (Figure 6D). Furthermore, treatment with the ER stress inhibitors (PBA or TUDCA) or JNK inhibitor II prevented the elevation of hepatic Nlrp3, Casp1, and IL1b mRNA expression in ethanol-treated mice (Figure 6E). More importantly, serum levels of mature IL-1 $\beta$ protein were significantly elevated after E10d + 1B challenge, and such elevation was prevented with PBA or JNK inhibitor II treatment (Figure 6F).

To test whether caspase-1 contributes to ER stress-mediated induction of particle release, the caspase-1 inhibitor Ac-YVAD-amk was used. As illustrated in Figure 6, G-K, treatment with Ac-YVAD-amk markedly reduced serum particle number, mtDNA contents, serum ALT, and circulating neutrophils after E10d + 1B feeding compared with those with vehicle treatment. Treatment with Ac-YVAD-amk also slightly reduced the expression of Chop and Gadd34, but not Ero1a, Ero1b, Pdi, and Grp78 (Supplemental Figure 3). 


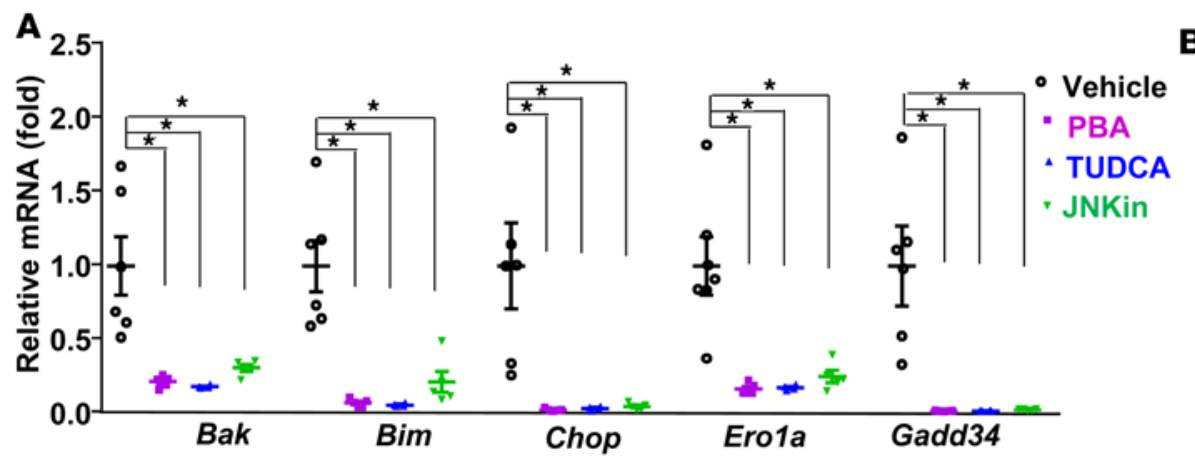

B
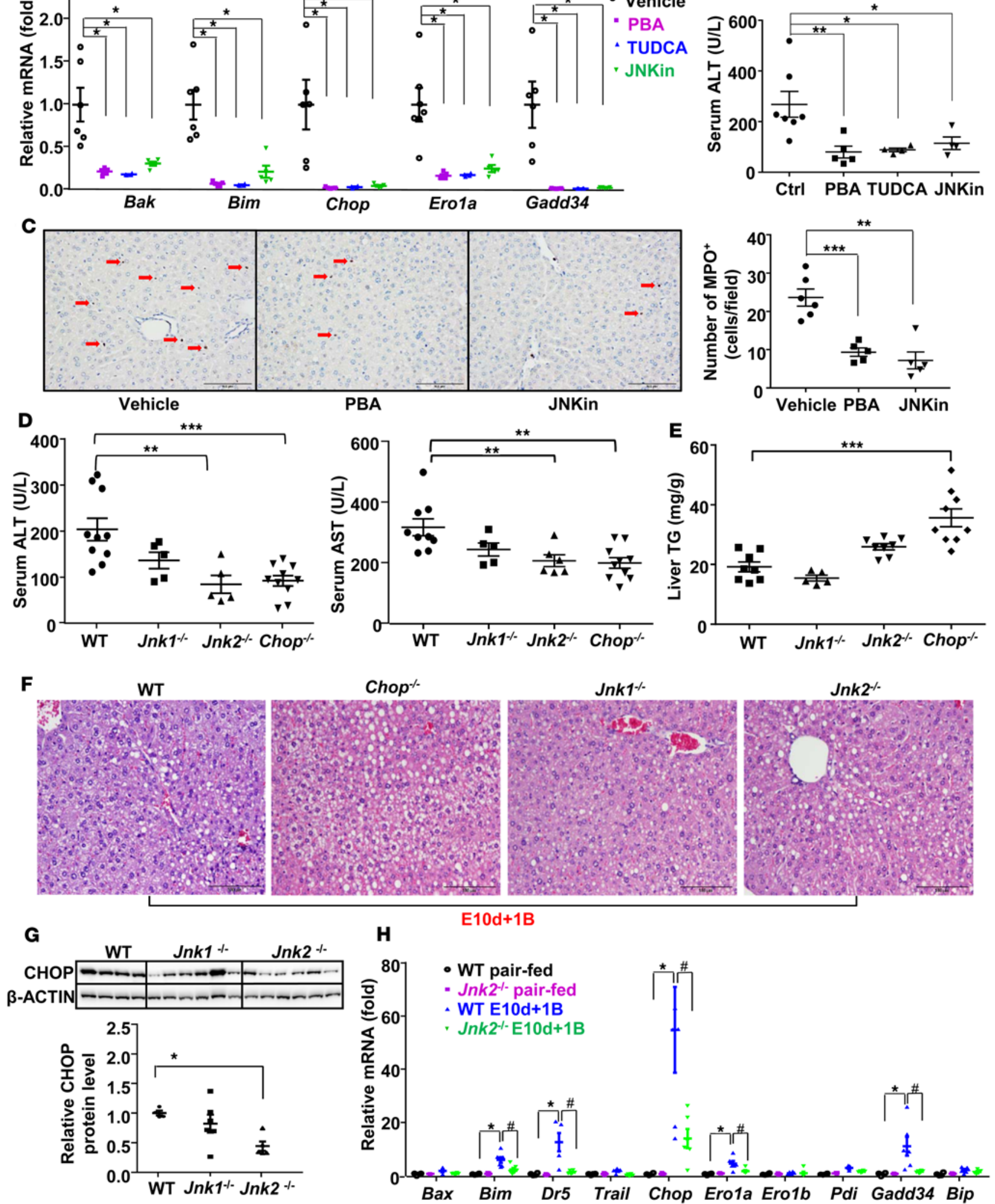

Figure 4. E10d + 1B-activated JNK2 and ER stress contribute to liver injury. (A-C) C57BL/6) mice were subjected to E10d + 1B feeding and received i.p. injection of ER stress inhibitors (PBA at $1 \mathrm{mg} / \mathrm{kg}$ and TUDCA at $500 \mathrm{mg} / \mathrm{kg}$ ) or JNK inhibitor II (JNKin, $20 \mathrm{mg} / \mathrm{kg}$ ) $15 \mathrm{~min}$ before gavage. Mice were euthanized at 9 hours after gavage ( $n=4-7$ per group). (A) Hepatic mRNA levels of ER stress-related genes were analyzed by real-time PCR; (B) serum ALT levels were measured; and $(\mathbf{C})$ representative MPO staining images $(\times 200)$ and quantification of MPO+ cells per field $(\times 100)$ in the liver are 


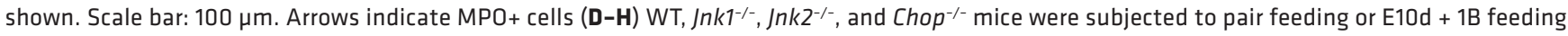
and were euthanized at 9 hours after gavage. (D) Serum ALT and AST levels were measured $(n=5-10)$; $(\mathbf{E})$ liver triglyceride content was measured ( $n=$ 5-9); (F) representative H\&E staining images $(\times 200)$ are shown; (G) Western blot analyses of CHOP and $\beta$-actin in the liver (upper panel) and the corresponding quantification data (lower panel) are shown ( $n=4-6$ per group); and (H) ER stress-related genes in the liver were analyzed by real-time PCR ( $n=5-6$ per group). Ordinary 1-way ANOVA with Tukey's test was performed. ${ }^{*} P<0.05,{ }^{*} P<0.01,{ }^{*}{ }^{*} P<0.001,{ }^{*} P<0.05$.

Hepatocytes are a source of elevated mtDNA in MPs after E10d $+1 B$ ethanol challenge. Next, we tested whether the increased mtDNA-contained MPs derived from hepatocytes after ethanol treatment by performing 3 experiments. First, serum microparticles were isolated and subjected to Western blot for analyses of CYP2E1 and $\beta$-actin. The results in Figure 7A show that CYP2E1 protein was detected in the MPs from E10d + 1B mice but not in those from pair-fed mice. Second, primary hepatocytes were treated with ethanol or ER stress inducer thapsigargin, followed by measurement of Cyto c ox DNA levels in the supernatant MPs. As illustrated in Figure 7B, Cyto c ox DNA levels in the supernatant MPs were significantly higher in ethanol- or thapsigargin-treated groups than in control groups. Lastly, hepatocyte-specific Perk-KO mice had significantly lower serum levels of MP mtDNA contents, ALT, and circulating neutrophils after E10d + 1B feeding compared with the WT mice (Figure 7, C-F). Taken together, our data confirmed that hepatocytes are the source of increased mtDNA in MPs.

MPs isolated from sera of E10d +1B-treated mice induce neutrophilia in chronically ethanol-fed mice via a TLR9-dependent mechanism. Previous studies reported that mtDNA promotes neutrophil infiltration via the activation of TLR9 (26) and that activation of TLR9 contributes to E10d + 1B ethanol-induced liver injury and inflammation (13). We next asked whether mtDNA in particles from E10d + 1B-treated mice contributes to liver injury and elevation of circulating neutrophils. To test this hypothesis, we purified mtDNA-enriched MPs from the sera of pair-fed and E10d + 1B-treated mice, and we injected these MPs into chronically ethanol-fed mice (E10d). As illustrated in Figure 8A, injection of MPs from E10d $+1 B$-treated mice induced higher levels of total number of circulating neutrophils, monocytes, and lymphocytes in E10d-fed WT mice but not in E10d-fed Tlr $9^{-1-}$ mice (Figure 8B). Injection of MPs from E10d + 1B-treated mice did not significantly elevate serum ALT in E10d-fed WT mice compared with injection of MPs from pair-fed mice, although there was a trend increase (Figure $8 \mathrm{C}$ ). Injection of MPs from E10d $+1 \mathrm{~B}$ mice or from pairfed mice generated comparable levels of serum ALT in E10d-fed Tlr $9^{-1-}$ mice (Figure 8C).

\section{Discussion}

Previous studies have demonstrated that acute alcohol consumption induces neutrophilia in human subjects with EAU, as well as in chronically ethanol-fed mice (5), but the underlying mechanisms remain obscure. In this current study, we found that RD markedly elevated serum MPs that are rich in mtDNA and that their levels are positively correlated with the levels of circulating neutrophils in EAU + RD patients. As mtDNA is known to promote neutrophil recruitment (26), elevated mtDNAs in MPs likely contribute to neutrophilia in the EAU $+\mathrm{RD}$ group, as well as in the mouse model of E10d + 1B ethanol feeding. Mechanistically, we found that the release of mtDNA-enriched MPs is secondary to the activation of ER stress and its activation of the inflammasome. The schematic diagram illustrating the proposed mechanism is summarized in Figure 9.

The role of ER stress in the pathogenesis of alcohol-induced liver injury has been described, especially in a mouse model of chronic ethanol feeding (27-29). In our present study, we found that the activation of ER stress is much greater in the $\mathrm{E} 10 \mathrm{~d}+1 \mathrm{~B}$ ethanol model when compared with either chronic ethanol feeding (E10d) or binge (1B) model alone in mice. From the morphological standpoint using electron microscopy, we observed obvious ER dilation in the liver from E10d + 1B mice, suggesting that short-term chronic ethanol feeding sensitizes mice to binge drinking-induced ER stress activation. It is well known that chronic ethanol feeding upregulates hepatic CYP2E1 protein levels and that elevated CYP2E1 exacerbates hepatic ER stress $(35,36)$. Thus, it is plausible to speculate that short-term ethanol feeding elevated hepatic CYP2E1 levels, which make the liver become more susceptible to an additional ethanol binge-induced ER stress.

ER stress is known to induce liver injury via the induction of apoptosis (27-29). In the current study, we provided several lines of evidence suggesting a novel mechanism by which ER stress promotes liver injury via the induction of circulating neutrophils and hepatic neutrophil infiltration. First, genetic deletion of ER stress-related genes (e.g., Chop, Jnk2, Perk) markedly prevented E10d + 1B-induced elevation of serum ALT and AST, serum particles (MPs, exosomes), and activation of the caspase- 1 inflammasome. Second, a critical role of ER stress in E10d + 1B ethanol-induced neutrophilia and liver injury was also confirmed by the stud- 
A
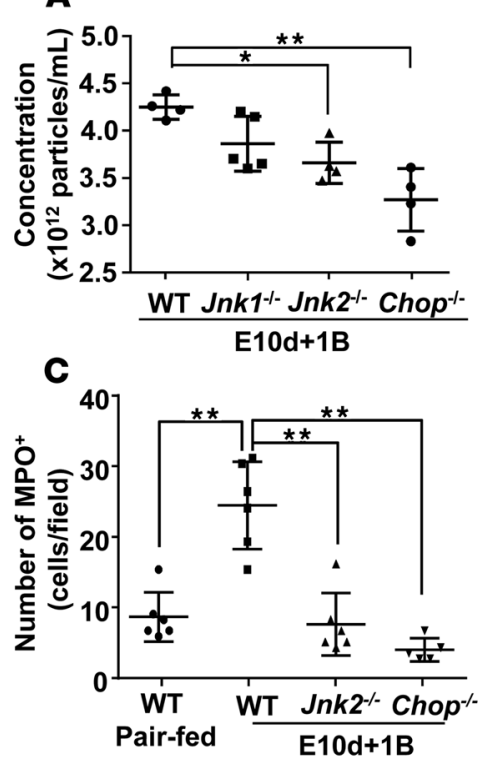

B

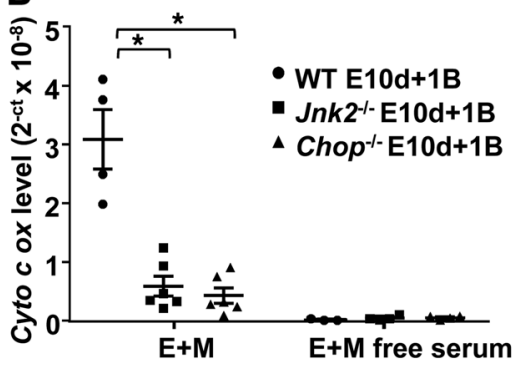

D

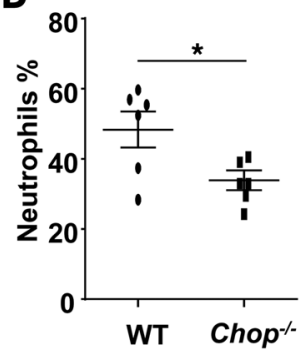

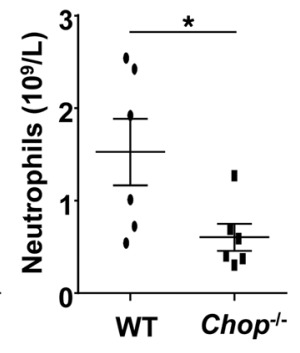

Figure 5. Elevations of serum particles, mtDNA, and hepatic neutrophils infiltration after E10d + 1B ethanol feeding are dependent on CHOP and JNIK2. WT, Jnk $1^{-1-}$, Jnk2 ${ }^{-/-}$, and Chop ${ }^{-/-}$mice were subjected to E10d + 1B feeding and were euthanized 9 hours after gavage. (A) Serum particle numbers were determined ( $n=4$ per group). (B) The contents of Cyto c ox DNA in serum exosomes and $\operatorname{MPs}(E+M)$ and $E+M$-free sera were measured $(n=4-6)$. (C) Quantification of $\mathrm{MPO}^{+}$cells per field $(\times 100)$ in the liver is shown ( $n=5-6$ per group). (D) The percentage and the number of peripheral neutrophils $(n=6)$. Ordinary 1-way 2-way ANOVA with Tukey's test was performed. ${ }^{*} P<$ $0.05,{ }^{* *} P<0.01$

ies using compounds that inhibit ER stress pathway. Third, blocking caspase- 1 activation prevented E10d $+1 \mathrm{~B}$ ethanol-induced elevation of MP release, indicating that ER stress-dependent caspase-1 activation contributes to MP release and subsequent neutrophilia and liver injury. However, the mechanisms by which activation of caspase-1 promotes MP releases

in hepatocytes remain unknown. A recent study suggests that activation of caspase-1 plays a critical role in promoting macrophages to release highly pro-coagulant thromboinflammatory MPs by degrading filamin and facilitating cell-surface actin exposure (2 important cascades required for MP generation and release) (34). Whether this mechanism also contributes to caspase-1-induced MP release in our E10d + 1B model required further studies. In addition, activation of the caspase apoptotic pathway (caspase-3 and caspase-8) promotes MP release in several types of cells, including neutrophils (37), adipocytes (38), and hepatocytes (21). It will be interesting to investigate whether caspase-1 activation of pyroptosis, a type of programed cell death that is different from apoptosis (39), also contributes to MP formation and release.

ER stress activated JNK, which plays an important role in the pathogenesis of liver diseases (31). JNK has several isoforms, including JNK1 and JNK2, both of which are expressed in hepatocytes and may exert different functions in the liver. For example, previous studies revealed that JNK1 prompted steatosis, while JNK2 inhibited hepatocyte death in mouse models of steatohepatitis induced by feeding a high-fat diet or methionine- and choline-deficient (MCD) diet $(40,41)$. In addition, it was reported that JNK1, but not JNK2, promoted liver cancer progression in a mouse model of chemical-induced liver carcinogenesis (42). In contrast, JNK2 plays a more critical role than JNK1 in inducing hepatocyte apoptosis in a model of Con A/D-galactosamine-induced liver injury (43). In the present studies, our data also suggest that JNK2, not JNK1, plays an important role in E10d + 1B ethanol-induced liver injury and inflammation. These discrepant data on JNK1 and JNK2 may be because of the different animal models used that may preferentially activate different isoforms of JNKs in the liver.

Circulating mtDNA levels were elevated and contributed to the pathogenesis of NASH by activating macrophages (18). It is possible that the elevation of mtDNA-enriched MPs in our E10d $+1 \mathrm{~B}$ model also promotes macrophage activation and likely hepatic inflammation. In addition, we provided further evidence suggesting that highly elevated mtDNA-enriched MPs are important in the pathogenesis of alcohol-induced liver injury via the activation of neutrophils. We found that injection of MPs, which were isolated from the sera of mice with the E10d + 1B model, can cause neutrophilia in mice chronically fed with ethanol, likely through its receptor, TLR9, as neutrophilia caused by injection of MPs was diminished in $\mathrm{Tlr}^{9^{-1}}$ mice. In addition to mtDNA, many other components in MPs produced by stressed hepatocytes such as CD40 ligand (21), CXCL10 (24), ceramide (30), and miRNAs (25) - have also been implicated in promoting hepatic inflammation. However, future studies are needed to determine whether these non-mtDNA components also play roles in the induction of neutrophils in our E10d $+1 \mathrm{~B}$ model.

In conclusion, chronic alcohol consumption with binge drinking results in an increase in the levels of circulating mtDNA-enriched MPs, as a consequent of ER stress activation. These mtDNA-enriched MPs can cause neutrophilia and promote liver injury. Targeting mtDNA-enriched MPs may be a novel and attractive strategy for the treatment of ALD through their regulation of neutrophil functions. 
A Pair fed $\triangle E 10 d+1 B 6 h$

- E10d+1B3h VE10d+1B9h

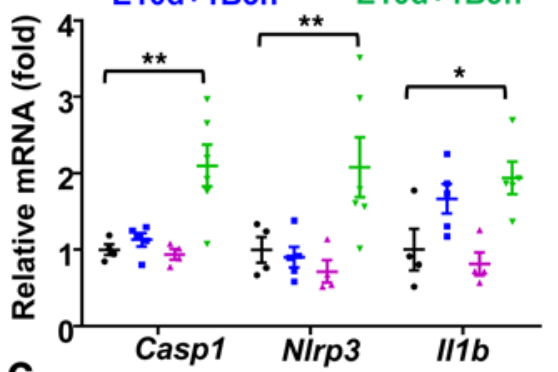

C

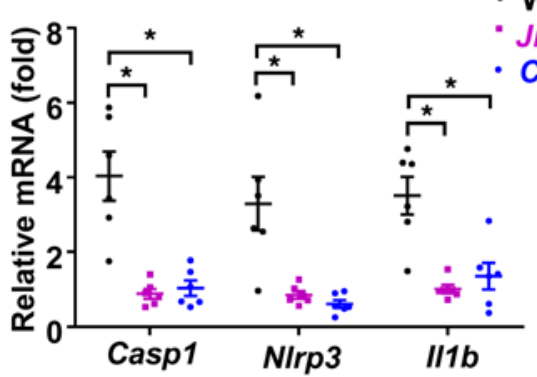

B

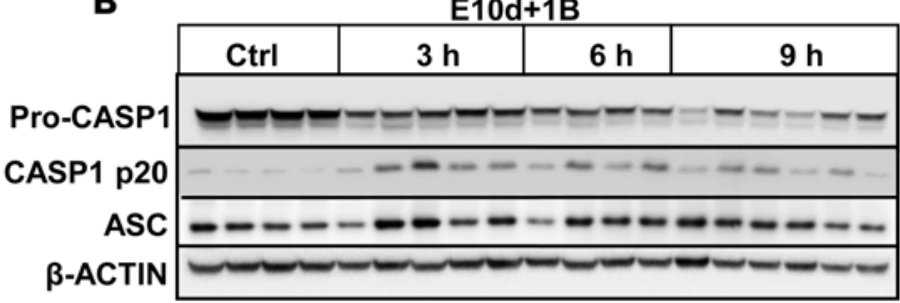

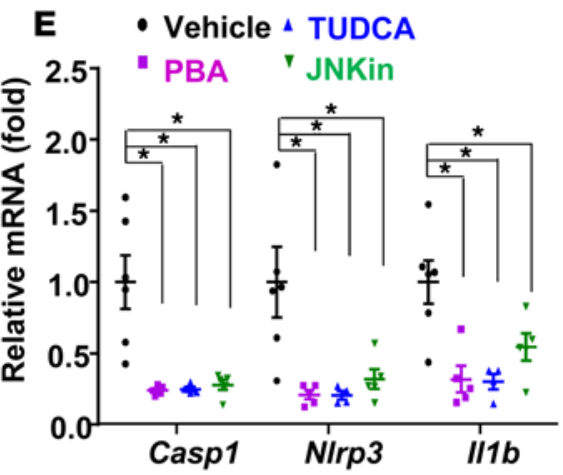

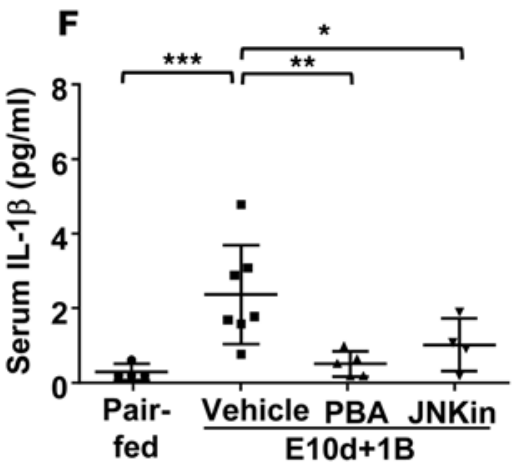

I

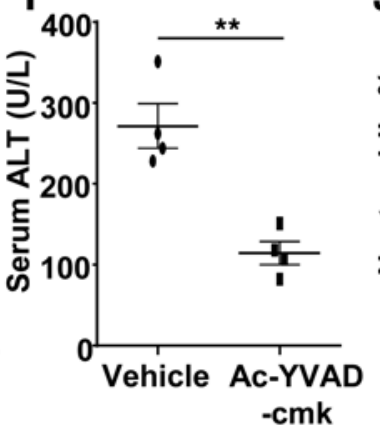

$-\mathrm{cmk}$

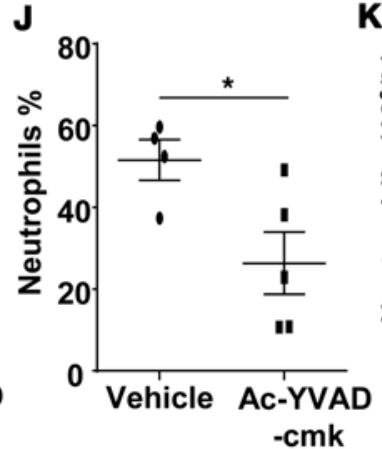

$E 10 d+1 B$

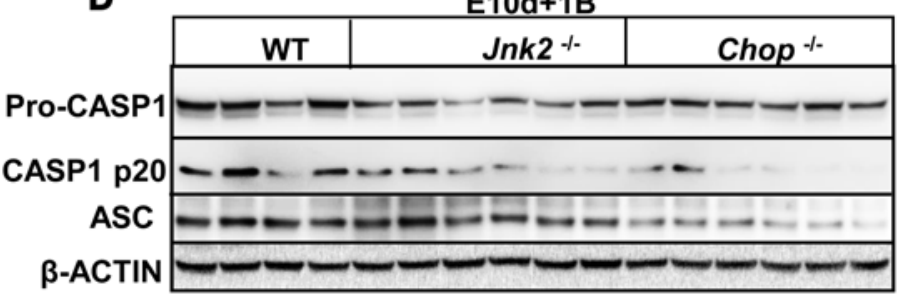

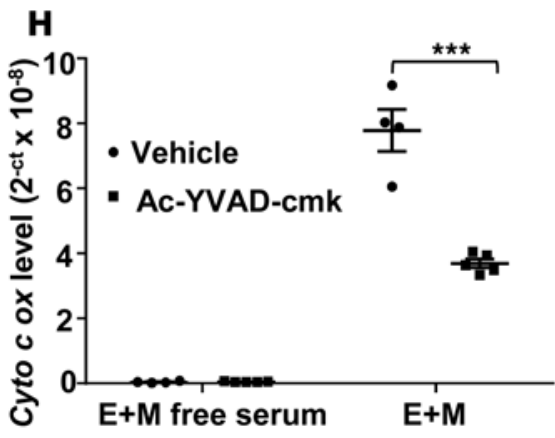

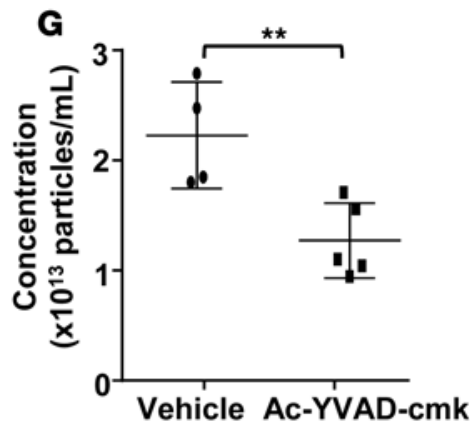

K

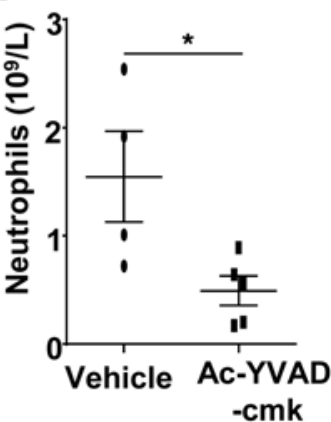

Figure 6. ER stress-dependent activation of caspase-1 contributes to E10d + 1B-induced elevation of particles and mtDNA contents in serum. (A) [57BL6] mice were subjected to E10d +1B feeding, and liver tissues were collected at 3 hours (h), $6 \mathrm{~h}$, and $9 \mathrm{~h}$ after gavage and subjected to real-time PCR analysis of inflammasome-related genes ( $n=4-6$ per group). (B) Western blot analyses of pro-CASP1, CASP1 p20, ASC, and $\beta$-actin in the liver

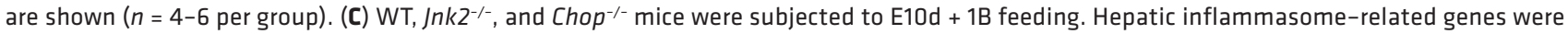
analyzed by real-time PCR. The value in pair-fed WT mice was set as $1(n=6)$. (D) Western blot analyses of pro-CASP1, CASP1 p20, ASC, and $\beta$-actin in the liver are shown $(n=4-6)$. (E and F) C57BL/6J mice were subjected to E10d $+1 B$ feeding and received ER stress inhibitors (PBA and TUDCA) and JNK inhibitor II 15 min before gavage $(n=4-6)$. Inflammasome-related genes were analyzed by real-time PCR $(E)$, and serum IL-1 $\beta$ was measured by ELISA (F). (G-K) E10d + 1B-treated mice were i.p. pretreated with vehicle or Ac-YVAD-cmk $(12.5 \mu \mathrm{mol} / \mathrm{kg}) 15 \mathrm{~min}$ before gavage $(n=4-5)$. (G) serum particle numbers, $(\mathbf{H})$ Cyto c ox DNA contents in serum $E+M$ and $E+M$-free sera, (I) serum ALT levels, and (J) the percentage and (K) number of peripheral neutrophils were determined. Ordinary 1-way ANOVA with Tukey's test was performed. ${ }^{*} P<0.05,{ }^{* *} P<0.01,{ }^{* *} P<0.001$. 

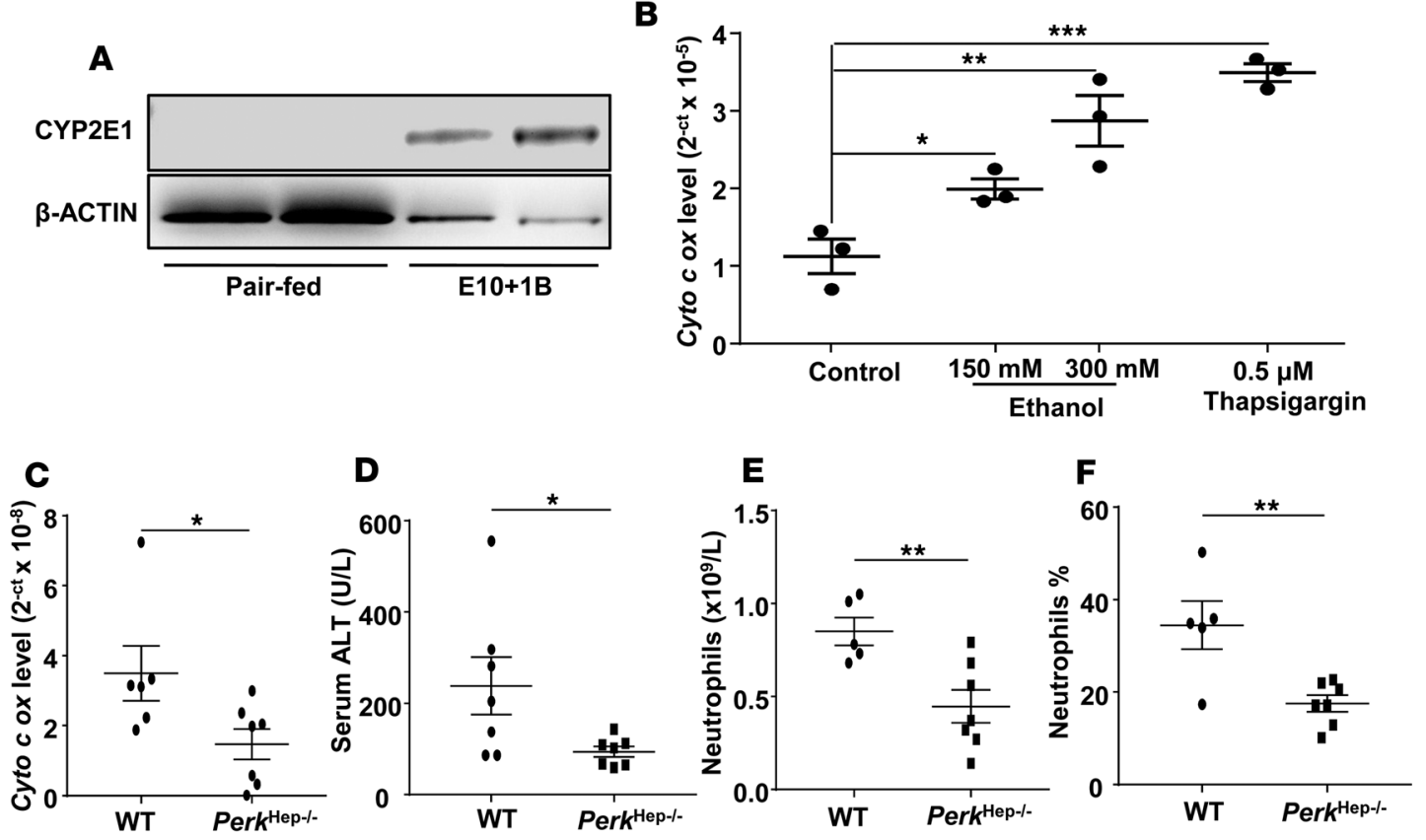

Figure 7. Hepatocytes are a source of increased mtDNA in MPs and contribute to neutrophilia. (A) Serum microparticles were isolated and subjected to Western blot; CYP2E1 and $\beta$-actin were detected. (B) Mouse hepatocytes were isolated and cultured, and they were treated with control, ethanol, and ER stress inducer thapsigargin for 3 hours. Cyto c ox DNA levels in the supernatant MPs per $1 \times 10^{6}$ hepatocytes were measured ( $n=3$ per group). (C-F) WT and hepatic Perk-KO (Perk ${ }^{\text {Hep }-l}-$ ) mice were subjected to E10d +1 B feeding ( $n=5-7$ per group). (C) Cyto c ox DNA contents in serum E + M, (D) serum ALT levels, (E) peripheral neutrophils, and (F) the percentage of peripheral neutrophils were measured. Ordinary 1-way ANOVA with Tukey's test was performed in B; unpaired Student $t$ test was performed in other panels. ${ }^{*} P<0.05,{ }^{* *} P<0.01,{ }^{* * *} P<0.001$.

\section{Methods}

Human subject cohort. The recruitment strategy of the human subject cohort was reported in our previous study (5). In brief, healthy controls were recruited from the clinics at the Roudebush Veterans Administration Medical Center, and patients with chronic EAU were from Fairbanks Drug and Alcohol Treatment Center (Indianapolis, Indiana, USA). Based on the information from the Time Line Follow-Back questionnaire (44), we dichotomized EAU subjects into those with and without RD (EAU + RD and EAU - RD, respectively), based on the history of alcohol consumption within the 10 days before the enrollment. For this study, a total of 201 subjects were included (30 healthy controls, 100 EAU + RD, and 71 EAU - RD).

Mice. Ten- to 12-week-old female C57BL/6J mice were used in this study. Mice were housed in a tem-

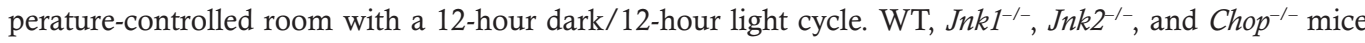
on a C57BL/6J background were purchased from The Jackson Laboratory and bred at the NIAAA animal facility. Perk ${ }^{\text {Hep-/ }}$ mice were obtained by crossing Perk floxed mice (The Jackson Laboratory) with Albumin Cre transgenic mice (The Jackson Laboratory) for several steps. Tlr9 ${ }^{-1-}$ mice on a C57BL/6 background were provided by Giorgio Trinchieri (National Cancer Institute, Rockville, Maryland, USA). All animals were cared for in accordance with the NIH guidelines.

E10d $+1 B$ ethanol feeding protocols. The E10d $+1 \mathrm{~B}$ feeding model was described previously (45). Briefly, 10- to 12-week-old female mice were initially fed a controlled Lieber-DeCarli liquid diet (catalog F1259SP, Bio-Serv) ad libitum for 5 days during acclimatization. Subsequently, the ethanol-fed groups were allowed free access to an ethanol diet (catalog F1258SP, Bio-Serv) containing 5\% (v/v) ethanol for 10 days. On day 11, they received a single dose of ethanol ( $5 \mathrm{~g} / \mathrm{kg}$ body weight) via oral gavage in the early morning and were sacrificed 3,6 , or 9 hours later. In addition, mice were fed an ethanol Lieber-DeCarli diet for 10 days as described for chronic feeding (designed as E10d) or received a single dose of ethanol ( $5 \mathrm{~g} / \mathrm{kg}$ body weight) via gavage in the early morning (designed as 1B) and were sacrificed 9 hours later. In some experiments, mice received ER stress inhibitors (PBA, catalog 8209860025, 1 mg/kg; TUDCA, catalog 580221, 500 mg/kg) (EMD Millipore) by i.p. injection, JNK inhibitor II (catalog 420119, $20 \mathrm{mg} / \mathrm{kg}$ ) (EMD Millipore) by i.m. injection, and caspase-1 inhibitor Ac-YVADcmk (Cayman Chemical, catalog 10014) by i.p. injection approximately 15 minutes before oral gavage. 


\title{
A E10d-fed WT mice
}
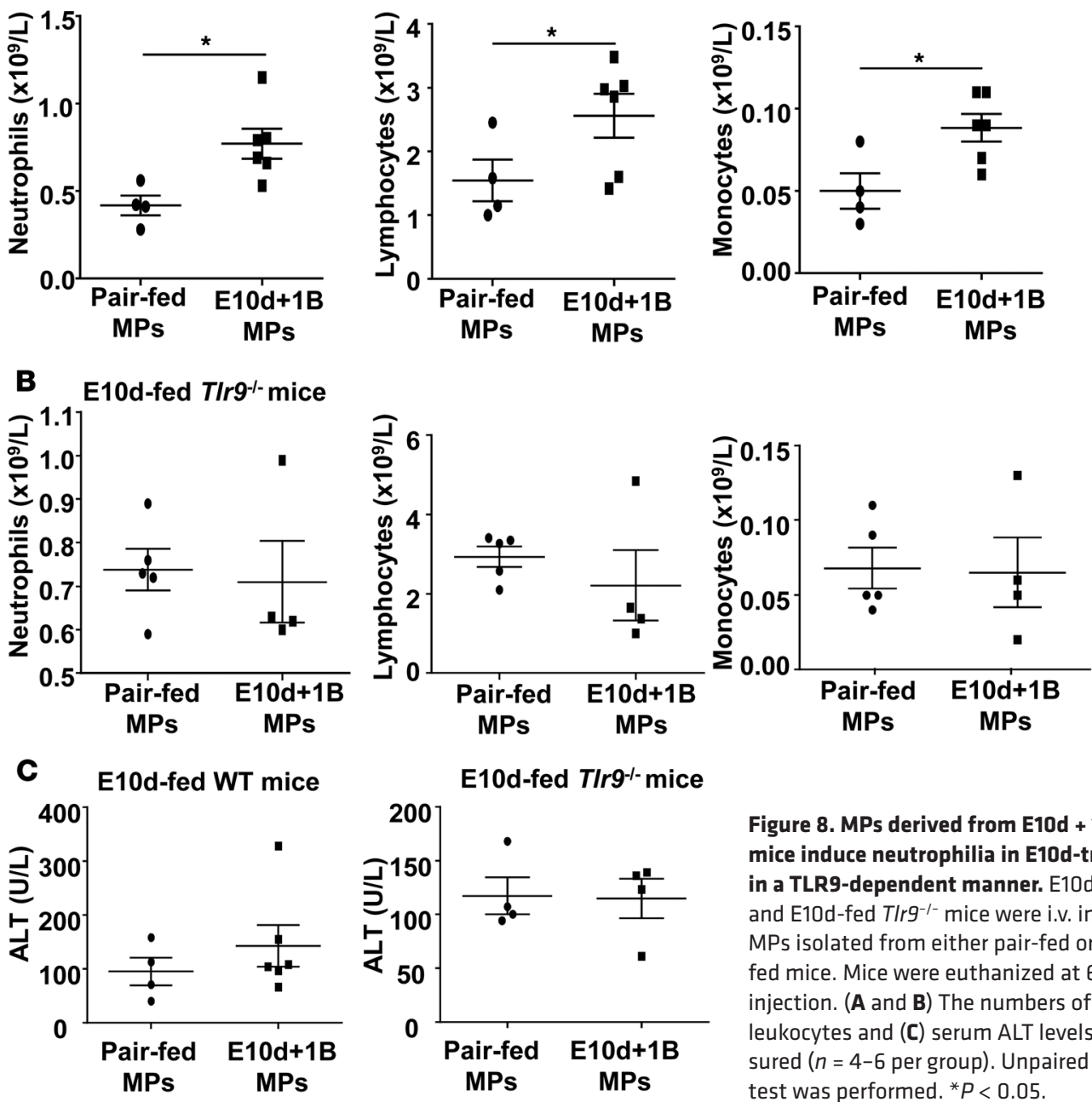

\begin{abstract}
Figure 8. MPs derived from E10d + 1B-treated mice induce neutrophilia in E10d-treated mice in a TLR9-dependent manner. E10d-fed WT and E10d-fed T/rg-/- mice were i.v. injected with MPs isolated from either pair-fed or E10d + 1Bfed mice. Mice were euthanized at 6 hours after injection. (A and $\mathbf{B}$ ) The numbers of peripheral leukocytes and (C) serum ALT levels were measured ( $n=4-6$ per group). Unpaired Student $t$ test was performed. ${ }^{*} P<0.05$.
\end{abstract}

Biochemical assays. Serum ALT and AST levels were determined by a Catalyst Dx chemistry analyzer (IDEXX Laboratories Inc.).

Liver histology and IHC. Liver specimens were fixed in 10\% buffered formalin and embedded in paraffin. Then, $4-\mu \mathrm{m}$ sections were used for IHC staining. IHC staining for myeloperoxidase (MPO) and CHOP were performed using a prediluted rabbit anti-MPO polyclonal antibody (catalog PP 023 AA, Biocare Medical LLC.) and anti-CHOP (catalog 5554, Cell Signaling Technology), respectively, followed by a rabbit ABC staining kit (catalog PK-6101, Vector Laboratories Inc.), according to the manufacturer's instructions. MPO+ cells were analyzed and quantified randomly from 10 microscopic fields at $100 \times$ magnification per sample.

Electron microscopy analyses of liver tissues. Liver tissues were fixed with $2 \%$ glutaraldehyde in $0.1 \mathrm{M}$ phosphate buffer ( $\mathrm{pH}$ 7.4) followed by $1 \% \mathrm{OsO}_{4}$. After dehydration, tissue sections were stained with uranyl acetate and lead citrate and were analyzed under a JEM 1016CX electron microscope (JEOL USA Inc.).

Quantitative PCR. Total cellular RNA was isolated from liver tissues using an RNeasy mini-kit (cata$\log 74104$, Qiagen). RNA (1 $\mu$ g of total) was reverse-transcribed by random primers and incubated with $200 \mathrm{U}$ of Moloney murine leukemia virus transcriptase at $37^{\circ} \mathrm{C}$ for 1 hour. The resulting single-stranded cDNA was then subjected to real-time PCR analyses with 18S rRNA as the internal control. Primer sequences are shown in Supplemental Table 1.

Western blotting. Tissues were homogenized in RIPA lysis buffer at $4^{\circ} \mathrm{C}$, vortexed, and centrifuged at $10,000 \mathrm{~g}$ at $4^{\circ} \mathrm{C}$ for 10 minutes. The supernatants were mixed in Laemmli loading buffer, boiled for 10 minutes, and then subjected to SDS-PAGE. After electrophoresis on $4 \%-12 \%$ Bis-Tris gel, proteins were transferred onto nitrocellulose membranes and blotted against respective primary antibodies overnight. Membranes were washed with $0.1 \%$ (v/v) Tween 20 in PBS (pH 7.4) and incubated with a 1:5,000 dilution 


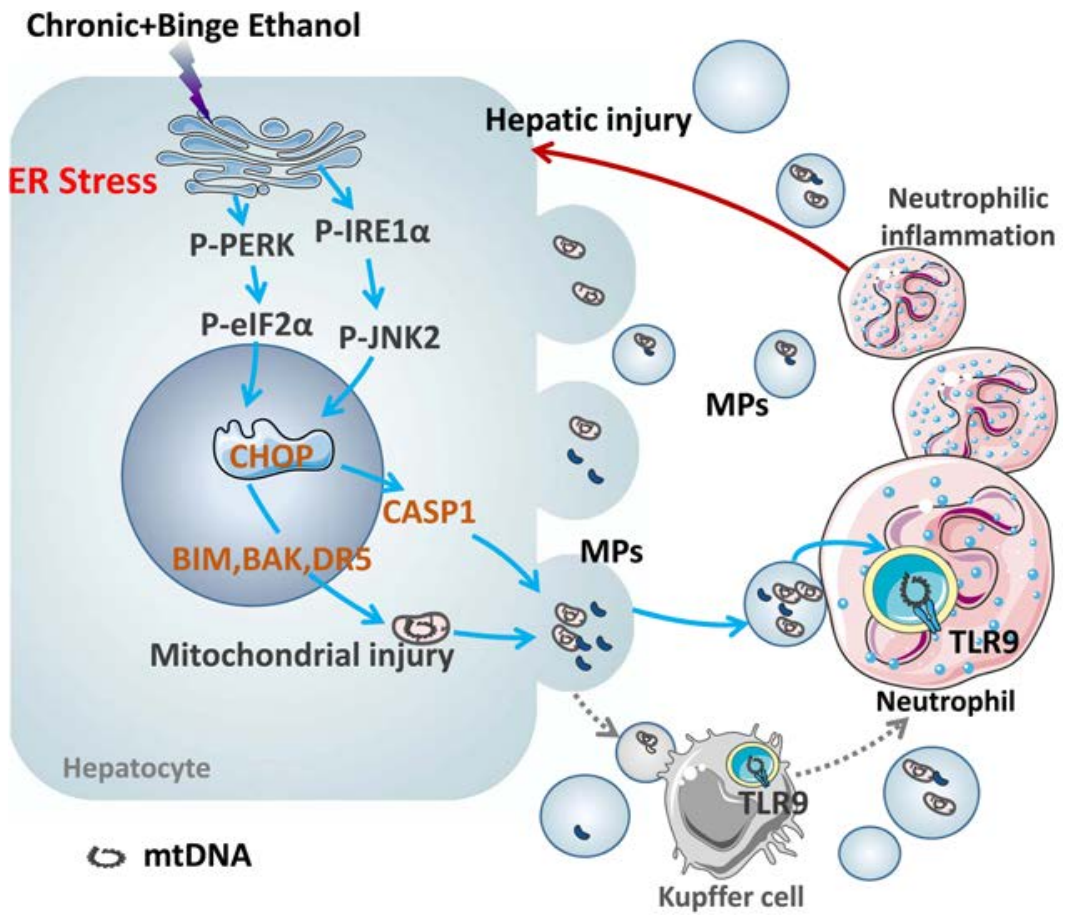

Figure 9. A model depicting the mechanisms by which chronic-plus-binge ethanol feeding induces neutrophilia. Chronic-plus-binge ethanol feeding induces circulating mtDNA-enriched MP release through hepatic ER stress and inflammasome activation. MtDNA-enriched MPs induce neutrophilia through their receptor, TLR9, thereby inducing liver inflammation and hepatocyte injury. Of note, Kupffer cells may also be a potential pathway contributing to mtDNA-enriched MP-induced neutrophilia.

of horseradish peroxidase-conjugated secondary antibody for 1 hour. Protein bands were visualized by SuperSignal West Femto Maximum Sensitivity Substrate (catalog 34095, Thermo Fisher Scientific).

Isolation and characterization of MPs, exosomes, and $E+M$ from human and mouse sera. Human sera were centrifuged at 3,000 $\mathrm{g}$ for 30 minutes to remove cell debris and then at $15,000 \mathrm{~g}$ for 30 minutes at $4^{\circ} \mathrm{C}$ to pellet MPs as previously described (18). The supernatant was added to the appropriate volume of ExoQuick Exosome precipitation solution (catalog EXOQ5A-1, System Biosciences). The exosomes were then isolated according to the ExoQuick kit protocol as described in the kit instruction.

In most mouse experiments, $\mathrm{E}+\mathrm{M}$ mixtures were isolated. Briefly, mouse sera were centrifuged at 3,000 $\mathrm{g}$ for 30 minutes to remove cell debris and then were added to the appropriate volume of ExoQuick Exosome precipitation solution. The $\mathrm{E}+\mathrm{M}$ mixtures were then isolated according to the ExoQuick kit protocol. The resulted supernatant was considered as $\mathrm{E}+\mathrm{M}$-free serum. In some mouse experiments, exosomes and MPs from mouse sera were also separately isolated by using the methods described above for isolation of these particles from human sera.

NTA. The concentration (particles/ml) and size distribution of EVs in mouse and human serum samples were analyzed using a Nanosight LM10 instrument with a $405 \mathrm{~nm}$ laser, NTA version 3.1 software (Malvern Instruments), and a constant flow rate syringe pump. A standard operating procedure file was used for each measurement, consisting of five 60 -second captures. Samples were diluted with sterile $1 \times$ PBS to obtain a measurable particle concentration between $5 \times 10^{7}$ and $5 \times 10^{9}$ particles $/ \mathrm{ml}$.

Injection of serum MPs and injection of MPs. Briefly, $1 \mathrm{ml}$ of serum mixture from E10d $+1 \mathrm{~B}$ or pair-fed mice were centrifuged at 3,000 $\mathrm{g}$ for 30 minutes to remove cell debris and then at $15,000 \mathrm{~g}$ for 30 minutes at $4^{\circ} \mathrm{C}$ to get MP pellets. The MP pellets ( $\sim \times 10^{12}$ particles) were resuspended in $150 \mu 1 \mathrm{PBS}$ and injected (i.v.) to the to the E10d WT or $\mathrm{Tlr}^{-/-}$mice.

DNA quantification. DNA was extracted from $200 \mu 1$ of plasma with QIAmp DNA mini kit (catalog 51306, Qiagen) according to the manufacturer's instructions, and qPCR was performed for the quantification of mtDNA using commercial primer. mtDNA was quantified using mouse Cyto $c$ ox primers (5'-ACCAAGGCCACCACACTCCT-3', 5'-ACGCTCAGAAGAATCCTGCAAAGAA-3') and human Cyto $c$ ox primers (5'-ATGACCCACCAATCACATGC-3', 5'-ATCACATGGCTAGGCCGGAG-3') as described previously $(26,46)$.

Cytokine measurement. Serum IL-1 $\beta$ levels were measured by a mouse ELISA Kit (catalog MLB00C, R\&D Systems).

Statistics. The results are expressed as the mean \pm SEM. All statistical analyses were performed using Prism 7 (GraphPad). Group comparisons were performed using the 2-tailed unpaired $t$ test or 1- or 2-way ANOVA, followed by Tukey's post-test as appropriate. $P<0.05$ was considered statistically significant.

Study approval. Animal care and experiments were conducted under the guidelines and protocols approved by the NIAAA Animal Care and Use Committee. The human studies were approved by the Indiana University Purdue University IRB, the Research and Development Committee of the Roudebush Veterans Administration Medical Center, and Fairbanks Drug and Alcohol Treatment Center. All participants provided written informed consent. 


\section{Author contributions}

YC and MJX designed and performed experiments, analyzed data, and wrote the paper. ZZ, WW, and HC conducted parts of experiments in mice, acquired data, and analyzed data. EHK, PSTY, RAR, RAS, and SL collected human serum samples, performed EV studies, and analyzed human data. BG obtained funding, supervised the project, designed experiments, analyzed data, and wrote the paper.

\section{Acknowledgments}

The authors are grateful to Wenxing Ding (University of Kansas, Kansas City, KS, USA) for electron microscopy analysis of liver tissues, and to the lab members for critical reading of the manuscript. This work was supported by the intramural program of NIAAA, NIH (BG); by the intramural program of NIDDK, NIH (RAS), and by grant 1I01CX000361-01 from the Veterans Affairs Research and Administration; by grant W81XWH-12-1-0497 from United States Department of Defense; and by R01 DK107682, R01AA025208, and R21AA024935 (SL).

Address correspondence to: Bin Gao, Laboratory of Liver Diseases, National Institute on Alcohol Abuse and Alcoholism, National Institutes of Health, 5625 Fishers Lane, Bethesda, Maryland, 20892, USA. Phone: 301.443.3998; Email: bgao@mail.nih.gov.

1. Gao B, Bataller R. Alcoholic liver disease: pathogenesis and new therapeutic targets. Gastroenterology. 2011;141(5):1572-1585.

2. Rehm J, Samokhvalov AV, Shield KD. Global burden of alcoholic liver diseases. J Hepatol. 2013;59(1):160-168.

3. Nagy LE, Ding WX, Cresci G, Saikia P, Shah VH. Linking Pathogenic Mechanisms of Alcoholic Liver Disease With Clinical Phenotypes. Gastroenterology. 2016;150(8):1756-1768.

4. Gao B, Tsukamoto H. Inflammation in Alcoholic and Nonalcoholic Fatty Liver Disease: Friend or Foe? Gastroenterology 2016;150(8):1704-1709.

5. Li M, et al. MicroRNA-223 ameliorates alcoholic liver injury by inhibiting the IL-6-p47(phox)-oxidative stress pathway in neutrophils. Gut. 2017;66(4):705-715.

6. Bertola A, Park O, Gao B. Chronic plus binge ethanol feeding synergistically induces neutrophil infiltration and liver injury in mice: a critical role for E-selectin. Hepatology. 2013;58(5):1814-1823.

7. Chang B, et al. Short- or long-term high-fat diet feeding plus acute ethanol binge synergistically induce acute liver injury in mice: an important role for CXCL1. Hepatology. 2015;62(4):1070-1085.

8. Lazaro R, et al. Osteopontin deficiency does not prevent but promotes alcoholic neutrophilic hepatitis in mice. Hepatology. 2015;61(1):129-140

9. Kobayashi Y. The role of chemokines in neutrophil biology. Front Biosci. 2008;13:2400-2407.

10. Bautista AP. Neutrophilic infiltration in alcoholic hepatitis. Alcohol. 2002;27(1):17-21.

11. Dominguez M, et al. Hepatic expression of CXC chemokines predicts portal hypertension and survival in patients with alcoholic hepatitis. Gastroenterology. 2009;136(5):1639-1650.

12. Sheron N, et al. Circulating and tissue levels of the neutrophil chemotaxin interleukin- 8 are elevated in severe acute alcoholic hepatitis, and tissue levels correlate with neutrophil infiltration. Hepatology. 1993;18(1):41-46.

13. Roh YS, Zhang B, Loomba R, Seki E. TLR2 and TLR9 contribute to alcohol-mediated liver injury through induction of CXCL1 and neutrophil infiltration. Am J Physiol Gastrointest Liver Physiol. 2015;309(1):G30-G41.

14. Wieser V, et al. Reversal of murine alcoholic steatohepatitis by pepducin-based functional blockade of interleukin- 8 receptors. Gut. 2017;66(5):930-938.

15. Robbins PD, Dorronsoro A, Booker CN. Regulation of chronic inflammatory and immune processes by extracellular vesicles. $J$ Clin Invest. 2016;126(4):1173-1180.

16. Huang-Doran I, Zhang CY, Vidal-Puig A. Extracellular Vesicles: Novel Mediators of Cell Communication In Metabolic Disease. Trends Endocrinol Metab. 2017;28(1):3-18.

17. Hirsova P, et al. Extracellular vesicles in liver pathobiology: Small particles with big impact. Hepatology. 2016;64(6):2219-2233

18. Garcia-Martinez I, et al. Hepatocyte mitochondrial DNA drives nonalcoholic steatohepatitis by activation of TLR9. JClin Invest. 2016;126(3):859-864.

19. Schattenberg JM, Lee MS. Extracellular Vesicles as Messengers Between Hepatocytes and Macrophages in Nonalcoholic Steatohepatitis. Gastroenterology. 2016;150(4):815-818.

20. Hirsova P, et al. Lipid-Induced Signaling Causes Release of Inflammatory Extracellular Vesicles From Hepatocytes. Gastroenterology. 2016;150(4):956-967.

21. Verma VK, et al. Alcohol stimulates macrophage activation through caspase-dependent hepatocyte derived release of CD40L containing extracellular vesicles. J Hepatol. 2016;64(3):651-660.

22. Saha B, Momen-Heravi F, Kodys K, Szabo G. MicroRNA Cargo of Extracellular Vesicles from Alcohol-exposed Monocytes Signals Naive Monocytes to Differentiate into M2 Macrophages. J Biol Chem. 2016;291(1):149-159.

23. Sato K, Meng F, Glaser S, Alpini G. Exosomes in liver pathology. J Hepatol. 2016;65(1):213-221.

24. Ibrahim SH, et al. Mixed lineage kinase 3 mediates release of C-X-C motif ligand 10-bearing chemotactic extracellular vesicles from lipotoxic hepatocytes. Hepatology. 2016;63(3):731-744.

25. Eguchi A, et al. Extracellular vesicles released by hepatocytes from gastric infusion model of alcoholic liver disease contain a MicroRNA barcode that can be detected in blood. Hepatology. 2017;65(2):475-490. 
26. Zhang Q, et al. Circulating mitochondrial DAMPs cause inflammatory responses to injury. Nature. 2010;464(7285):104-107. 27. Ji C. New Insights into the Pathogenesis of Alcohol-Induced ER Stress and Liver Diseases. Int J Hepatol. 2014;2014:513787.

28. Fernandez A, et al. ASMase is required for chronic alcohol induced hepatic endoplasmic reticulum stress and mitochondrial cholesterol loading. J Hepatol. 2013;59(4):805-813.

29. Dara L, Ji C, Kaplowitz N. The contribution of endoplasmic reticulum stress to liver diseases. Hepatology. 2011;53(5):1752-1763.

30. Kakazu E, Mauer AS, Yin M, Malhi H. Hepatocytes release ceramide-enriched pro-inflammatory extracellular vesicles in an IRE1 $\alpha$-dependent manner. J Lipid Res. 2016;57(2):233-245.

31. Seki E, Brenner DA, Karin M. A liver full of JNK: signaling in regulation of cell function and disease pathogenesis, and clinical approaches. Gastroenterology. 2012;143(2):307-320.

32. Tilg H, Moschen AR, Szabo G. Interleukin-1 and inflammasomes in alcoholic liver disease/acute alcoholic hepatitis and nonalcoholic fatty liver disease/nonalcoholic steatohepatitis. Hepatology. 2016;64(3):955-965.

33. Cui K, et al. Invariant NKT cells promote alcohol-induced steatohepatitis through interleukin-1 $\beta$ in mice. $J$ Hepatol. 2015;62(6):1311-1318.

34. Rothmeier AS, et al. Caspase-1-mediated pathway promotes generation of thromboinflammatory microparticles. J Clin Invest. 2015;125(4):1471-1484.

35. Lewis MD, Roberts BJ. Role of CYP2E1 activity in endoplasmic reticulum ubiquitination, proteasome association, and the unfolded protein response. Arch Biochem Biophys. 2005;436(2):237-245.

36. Dey A, Kessova IG, Cederbaum AI. Decreased protein and mRNA expression of ER stress proteins GRP78 and GRP94 in HepG2 cells over-expressing CYP2E1. Arch Biochem Biophys. 2006;447(2):155-166.

37. Midura EF, Prakash PS, Johnson BL, Rice TC, Kunz N, Caldwell CC. Impact of caspase- 8 and PKA in regulating neutrophil-derived microparticle generation. Biochem Biophys Res Commun. 2016;469(4):917-922.

38. Eguchi A, et al. Microparticles release by adipocytes act as "find-me" signals to promote macrophage migration. PLoS One. 2015;10(4):e0123110.

39. Miao EA, Rajan JV, Aderem A. Caspase-1-induced pyroptotic cell death. Immunol Rev. 2011;243(1):206-214.

40. Schattenberg JM, et al. JNK1 but not JNK2 promotes the development of steatohepatitis in mice. Hepatology. 2006;43(1):163-172.

41. Singh R, Wang Y, Xiang Y, Tanaka KE, Gaarde WA, Czaja MJ. Differential effects of JNK1 and JNK2 inhibition on murine steatohepatitis and insulin resistance. Hepatology. 2009;49(1):87-96.

42. Hui L, Zatloukal K, Scheuch H, Stepniak E, Wagner EF. Proliferation of human HCC cells and chemically induced mouse liver cancers requires JNK1-dependent p21 downregulation. J Clin Invest. 2008;118(12):3943-3953.

43. Ni HM, Chen X, Ding WX, Schuchmann M, Yin XM. Differential roles of JNK in ConA/GalN and ConA-induced liver injury in mice. Am J Pathol. 2008;173(4):962-972.

44. Sobell LC, Sobell MB. Timeline follow-back: A technique for assessing self-reported alcohol consumption. In: Allen J, Litten RZ, eds. Measuring Alcohol Consumption: Psychosocial and Biological Methods. Totowa, NJ: Humana Press;1992;41-72.

45. Bertola A, Mathews S, Ki SH, Wang H, Gao B. Mouse model of chronic and binge ethanol feeding (the NIAAA model). Nat Protoc. 2013;8(3):627-637.

46. McGill MR, Sharpe MR, Williams CD, Taha M, Curry SC, Jaeschke H. The mechanism underlying acetaminophen-induced hepatotoxicity in humans and mice involves mitochondrial damage and nuclear DNA fragmentation. J Clin Invest. 2012;122(4):1574-1583 